\title{
Appropriate monitoring of oral nutritional supplementation: An audit within a large GP practice
}

\author{
A. S. Tahim and D. O'Gallagher \\ Park Medical Centre, Inver Court, Invermead Close, London, W6 OQG, UK
}

\begin{abstract}
Malnutrition affects over 2 million people in the UK but remains under-recognised and under-treated ${ }^{(1)}$. While much work has evaluated the indications for nutritional supplementation, there is relatively little investigating how accurately the effects of such interventions are monitoring after they are prescribed ${ }^{(2)}$. NICE guidelines suggest that patients receiving oral nutritional supplementation should be monitored on a 3-6 month basis, using a number of potential observations or measurements such as Body Mass Index (BMI) ${ }^{(3)}$. Numerous other tools highlight BMI as a useful and practical measure of malnutrition ${ }^{(4)}$. As such, our audit was conducted to assess whether there was appropriate documentation prior to starting and stopping oral supplementation and whether BMI was measured within 3 months of starting treatment.

All senior doctors at the practice were asked to complete a short survey identifying the nutritional supplements that they have previously prescribed. The results were collated and patients who had been prescribed any of these supplements were retrospectively identified over a one year period. Electronic patient records were analysed, investigating indication, BMI records prior to treatment and appropriate subsequent monitoring. 39 patients were prescribed supplements. $64.1 \%$ of all treatments were initiated at the practice. Of these, the reason for treatment was documented in $68.0 \%$. BMI was recorded prior to treatment in $51.3 \%$ of all cases. The BMI was monitored within 3 months of treatment starting in $10.0 \%$ of cases. BMI was recorded within 3 months of the most recent prescription in $20.5 \% .18$ patients within the study $(46.2 \%)$ were having ongoing treatment. Of the $53.8 \%$ that had their supplementation stopped, none had a documented reason. Overall, a total of $2.6 \%$ patients had or are having their treatment appropriately monitored.

Our results show that monitoring of oral nutritional supplementation does not meet the standards recommended in recent NICE guidelines. Results were circulated to senior doctors and a number of potential future developments were outlined. These included local practice guidelines for monitoring of supplements, changes to computer systems to facilitate monitoring at appropriate intervals and educational sessions for staff to encourage ongoing assessment of response to oral supplementation. Once changes have been implemented, the effects are to be assessed by a repeat analysis of the monitoring of oral supplements.

In conclusion, this audit shows that while prescribing oral nutritional supplements is relatively commonplace, guidelines outlining how such treatment should be monitored are poorly adhered to. This study can form the basis on which changes can be made at local levels to improve the standards of the monitoring of prescribed oral nutritional supplementation.
\end{abstract}

1. Malnutrition Advisory Group, THE 'MUST' REPORT - Nutritional screening of adults: A multidisciplinary responsibility. 2010.

2. Gall, MJ, JE Harmer and HJ Wanstall, Prescribing of oral nutritional supplements in Primary Care: can guidelines supported by education improve prescribing practice? Clinical nutrition (Edinburgh, Scotland), 2001. 20(6): p. 511-515.

3. NICE, CG32 Nutrition support in adults. 2006.

4. Malnutrition Advisory Group, Malnutrition Universal Screening Tool. 2003. 